\title{
Clinical Research on the Postoperative Recurrence of Non Muscle-Invasive Bladder Cancer
}

\author{
Hui Xu, Chang-Li Xu, Jin-Qi Song, Ya-Nan Zhou \\ Department of Urology, The Affiliated Hospital of Cheng de Medical University, Cheng de, PR of China.
}

\begin{abstract}
Background: Bladder cancer is a common malignant tumor in the urinary system, in which non muscle-invasive bladder cancer (NMIBC) is the most common. The recurrence rate of the patients after operation is high, which has a serious impact on the physical and mental health of the patients. Therefore, how to reduce the recurrence rate of NMIBC patients is the focus of clinical research.

Objectives: To study the recurrent factor of NMIBC after surgery for promotion of therapeutic efficacy.

Methods: Retrospective analysis of one hundred patients of carcinoma of urinary bladder were consecutively enrolled and data on gender, age, tumor stage, tumor grade, tumor size, tumor configuration, multiplicity, date of surgery, tumor histology, recurrence history, resident area, frequency of tumor recurrence, follow-up history as screening target to analyze by Cox proportional-risk model, and screen dependent factor which can be used to predict tumor recurrent risk after surgery.

Results: Tumor size, tumor stage, tumor grade, frequency of tumor recurrence, multiplicity and history of regular follow-up are related to NMIBC recurrence after surgery.

Conclusion: Aggregate analysis of these factors can evaluate recurrent risk of NMIBC and it is significant to the therapy of NMIBC.
\end{abstract}

Keywords: NMIBC, recurrence, multivariate analysis.

\section{Introduction}

Bladder cancer is a most common malignant tumor in genitourinary system. These can be divided into Non muscle invasive (NMIBC) and muscle invasive bladder cancer. NMIBC accounts for $75 \% \sim 85 \%$ of the total bladder cancer. ${ }^{1}$ According to relevant research statistics, the recurrence rate of NMIBC after operation is as high as $60 \% \sim 70 \%$, of which about $20 \% \sim 30 \%$ recurrence of tumor will develop into muscle invasive and metastatic disease. This study sheds light on factors associated to the recurrence of NMIBC after operation and can suggest early aggressive treatment.

\section{Materials \& Methods}

Materials: Retrospective analysis of case notes of 100 patients with NMIBC, those were confirmed by pathology after surgery in the Affiliated Hospital of Chengde Medical University from May 2010 to May 2018 was carried. The age of the patients ranged from 42 to 77 years old (average age 55.4 \pm 3.4 ). There were 76 male patients and 24 female patients. Disease wise, single tumors were present in 65 cases and multiple tumors were present in 35 cases. Among these patients, 68 cases were primary tumors and 32 cases were recurrent tumors. The size of the tumors were from 0.3 to $3.5 \mathrm{~cm}$ with average size of $(2.15 \pm 0.56) \mathrm{cm}$. The operative methods included trans urethral resection of bladder tumor(TURBT) in 87 cases and partial resection of bladder in 10 cases, partial resection and ureteric re-implantation in 3 cases. Induction with immediate intra-vesical infusion of chemotherapeutic agent $30 \mathrm{mg}$ hydroxy-camptothecin(HCPT)(product of Huangshi Feiyun Pharmaceutical Limited Company,China) was done. The drug was added to $30 \mathrm{ml}$ normal saline for intra vesicle installation. This was followed by weekly installation of same for 8 weeks. This was consolidated with same dose once in a month for 10 consecutive months. Cystoscopy was examined every 3 months after operation for a total of 2 years.

The clinical stage of tumor was Ta stage in 35 cases, T1 stage in 52 cases and T2 stage in 13 cases. The pathological grade was G1 grade in 37 cases, G2 grade in 54 cases and G3 grade in 9 cases. All patients were followed up after surgery. The follow-up time was $3 \sim 96$ months, with an 
average time of 25 months. Altogether, 23 patient's had tumor recurrence during follow-up.

The exclusion criteria for intravesical installation of chemotherapy were: 1 . obvious postoperative hematuria, 2. those who suffered severe chemical cystitis and who were installed another chemotherapy drugs, 3. Patients who were not compliant to the treatment schedule of reexamination.

Methods: With gender, age, tumor grade, tumor stage, tumor types (primary or recurrent), tumor shape, size (was limited to $3 \mathrm{~cm}$ ), location of tumor (trigone, bladder neck, lateral walls, posterior wall, dome), multiplicity (single or multiple, multiple was limited to 8), Pathological type (transitional epithelial carcinoma, adenocarcinoma, squamous carcinoma), Type of operation (TURBT, partial resection of bladder, partial resection and bladder and ureteric re-implantation), frequency of tumor recurrence (was limited to once per year) and regular cystoscopic follow-up after surgery or not. Thus a total of 13 factors were used as indicators, and quantifies (assignment), as shown in table 1.

Table 1: Quantification of patients related factors influencing postoperative recurrence of NMIBC

\begin{tabular}{|c|c|c|c|c|c|c|c|c|c|c|}
\hline 氖 & 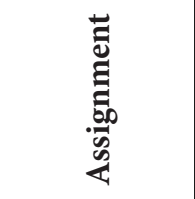 & 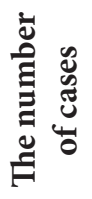 & 芦 & 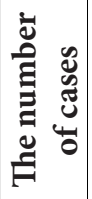 & 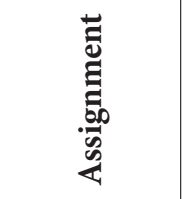 & 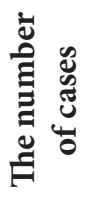 & 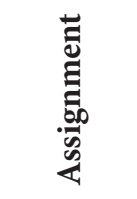 & 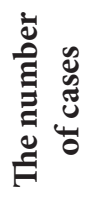 & 离 & 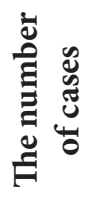 \\
\hline & 1 & & 2 & & 3 & & 4 & & 5 & \\
\hline Gender & Male & 76 & Female & 24 & & & & & & \\
\hline Age & $<60$ years old & 64 & $\geq 60$ years old & 36 & & & & & & \\
\hline Tumor grade & $\mathrm{G}_{1}$ & 37 & $\mathrm{G}_{2}$ & 54 & $\mathrm{G}_{3}$ & 9 & & & & \\
\hline Tumor stage & $\mathrm{T}_{\mathrm{is}}$ & 0 & $\mathrm{~T}_{\mathrm{a}}^{2}$ & 35 & $\mathrm{~T}_{1}^{3}$ & 52 & $\mathrm{~T} 2$ & 13 & & \\
\hline Tumor sources & Primary & 68 & Recurrent & 32 & & & & & & \\
\hline Tumor shape & Papillary & 25 & Crumb & 42 & Coralline & 33 & & & & \\
\hline Tumor size & $<3 \mathrm{~cm}$ & 57 & $\geq 3 \mathrm{~cm}$ & 43 & & & & & & \\
\hline Tumor location & $\begin{array}{c}\text { Cervical } \\
\text { mouth }\end{array}$ & 16 & Triangle & 35 & Back wall & 24 & $\begin{array}{c}\text { Two side } \\
\text { wall }\end{array}$ & 20 & $\begin{array}{c}\text { Top front } \\
\text { wall }\end{array}$ & 5 \\
\hline Tumor multiple & Single & 65 & Multiple $\geq 8$ & 5 & Multiple $<8$ & 30 & & & & \\
\hline $\begin{array}{l}\text { Organizational } \\
\text { sources }\end{array}$ & $\begin{array}{c}\text { Transitional } \\
\text { epithelial } \\
\text { carcinoma }\end{array}$ & 89 & Adenocarcinoma & 6 & $\begin{array}{l}\text { Squamous } \\
\text { carcinoma }\end{array}$ & 5 & & & & \\
\hline operation & TURBT & 87 & $\begin{array}{l}\text { Partial resection of } \\
\text { bladder }\end{array}$ & 10 & $\begin{array}{l}\text { Bladder partial } \\
\text { resection and } \\
\text { bladder ureter } \\
\text { reimplantation }\end{array}$ & 3 & & & & \\
\hline $\begin{array}{l}\text { Tumor } \\
\text { recurrence } \\
\text { frequency }\end{array}$ & $\begin{array}{c}\text { Once per } \\
\text { year }\end{array}$ & 6 & Twice per year & 22 & $\begin{array}{l}\text { Thrice per } \\
\text { year }\end{array}$ & 35 & & & & \\
\hline $\begin{array}{l}\text { Regular follow- } \\
\text { up after surgery }\end{array}$ & Yes & 100 & No & 0 & & & & & & \\
\hline
\end{tabular}

Statistical analysis: Cox proportional-risk model in SPSS 13.0 statistical software was selected for statistical analysis. In other words, the assignment of candidate indicators was respectively carried into the Cox proportional-risk model, and the Backward Stepwise method was adopted to screen the risk factors of postoperative recurrence of NMIBC by referring to the Likelihood Ratio. $P<0.05$, indicating statistical significance.

\section{Results}

A statistical test was conducted to determine whether all the regression coefficients of all candidate factors were 0 , and the statistics $x^{2}=25.470$, degree of freedom $u=10$, and $P<0.01$, indicating statistical significance, that at least one candidate factor was associated with postoperative recurrence of NMIBC. And then you went back and you got Cox Stepwise proportional-risk regression analysis 
table, as shown in table 2.

Table 2: Multivariate Cox stepwise proportional-risk regression analysis of postoperative recurrence of NMIBC

\begin{tabular}{|l|c|c|c|c|}
\hline & $\mathbf{N}$ & $\mathbf{R C}$ & $\mathbf{R R}(\mathbf{9 5} \% \mathbf{C I})$ & $\mathbf{P}$ \\
\hline Tumor multiplicity & 100 & 1.823 & $\begin{array}{c}5.823 \\
(2.462-12.746)\end{array}$ & $<0.001$ \\
\hline Tumor size & 100 & -0.562 & $\begin{array}{c}0.354 \\
(0.182-0.845)\end{array}$ & 0.013 \\
\hline Tumor stage & 100 & -2.384 & $\begin{array}{c}0.064 \\
(0.007-0.524)\end{array}$ & 0.010 \\
\hline Tumor grade & 100 & 1.745 & $\begin{array}{c}6.065 \\
(1.024-25.842)\end{array}$ & 0.046 \\
\hline $\begin{array}{l}\text { Tumor recurrence } \\
\text { frequency }\end{array}$ & 100 & 1.004 & $\begin{array}{c}2.776 \\
(0.914-8.145)\end{array}$ & 0.047 \\
\hline $\begin{array}{l}\text { Regular follow-up } \\
\text { after surgery }\end{array}$ & 100 & -2.234 & $\begin{array}{c}0.452 \\
(0.243-0.934)\end{array}$ & 0.018 \\
\hline
\end{tabular}

$\mathrm{N}=$ the number of cases, $\mathrm{RC}=$ Regression coefficient, $\mathrm{RR}(\mathbf{9 5 \%} \mathrm{CI})=$ relative risk (confidence interval), $P=P$ value

The results showed that six indicators, including multiplicity of tumor, tumor size, tumor stage, tumor grade, frequency of tumor recurrence, and regular followup after surgery, were directly related to postoperative recurrence of NMIBC. There was no correlation between gender, age, tumor shape, tumor sources, tumor location, organizational sources and operation.

\section{Discussion}

NMIBC had been described as superficial bladder cancer. Previous literature reports had shown that the postoperative local recurrence rate of NMIBC patients was relatively high, of which $50 \%$ recurred in the first year after surgery and about $2 / 3$ recurred in the second year after surgery. ${ }^{2}$ Some of the patients were accompanied by increased infiltration or malignant transformation, which was mainly caused by tumor growth caused by multiple foci or centroid growth. During surgery, some of the tumor cells fall off and can be implanted or retained, and recurrence can occur. How to predict and evaluate the risk of recurrence of NMIBC and choose appropriate treatment measures is of great significance to the treatment of clinical NMIBC.

At present, significant achievements had been made in predicting and evaluating the risk of tumor recurrence by using such indicators as tumor stage, tumor grade, tumor size, multi-focality of tumor, tumor location, tumor shape and frequency of tumor recurrence. European Association of Urology recommended tumor risk factor score is based on multiplicity of tumor, tumor size, tumor stage, tumor grade, frequency of tumor recurrence and other basic characteristics of bladder tumors, and determines the prognosis of bladder tumors by assigning different weights to the basic characteristics of these tumors.

This study showed that multiplicity of tumor multiple, tumor size, tumor stage, tumor grade, tumor recurrence frequency, and regular follow-up after surgery all played a role in postoperative survival (NMIBC recurrence). NMIBC has a high postoperative recurrence rate after TURBT, and a small number of patients may even develop muscle invasive bladder cancer. The commonly selected treatment in clinical practice is bladder perfusion assisted surgery, which can not only eliminate all the residual tumor cells, but also significantly reduce the postoperative recurrence rate, so as to prevent tumor recurrence. Therefore, all NMIBC patients are recommended for postoperative adjuvant bladder perfusion therapy, including bladder perfusion chemotherapy and bladder perfusion immunotherapy. Long-term studies have found that postoperative recurrence of bladder cancer is the main reason for the high mortality rate of bladder cancer patients. Several clinical studies reported in recent years have shown that regular infusion of chemotherapy drugs into the bladder after bladder cancer surgery can completely kill cancer cells and reduce the risk of tumor recurrence. . $^{3-5}$

Sylvester compared the recurrence rate of 2278 NMIBC patients with immediate post-TURBT intra-vesical installation chemotherapy and simple surgery through systematic review and meta-analysis. The five-year recurrence rate dropped from $58.8 \%$ to $44.8 \%{ }^{6}$ Since postoperative recurrence of bladder tumor is closely related to residual disease and ablative implantation during surgery, it is an effective measure to reduce tumor recurrence rate to kill residual tumor cells before they implant themselves. It has been reported that intra-vesical instillation drugs can reduce the short-term recurrence rate of NMIBC after TURBT by $15 \%-20 \%$ and the long-term recurrence rate by about $6 \% .^{7}$ Immediate bladder perfusion chemotherapy after TURBT can significantly reduce the recurrence rate 
of NMIBC, the principle of which is that immediate postoperative instillation chemotherapy can kill tumor cells disseminated in surgery and residual tumor cells on wound surface. In order to prevent tumor cell implantation, bladder perfusion chemotherapy should be completed within 24 hours after surgery. ${ }^{8-9}$ Early postoperative perfusion chemotherapy is recommended, for example, in the operating room or in the recovery room to achieve the best results. ${ }^{10}$ All NMIBC are recommended for immediate postoperative bladder instillation chemotherapy, but it is not recommended in the presence of bladder perforation during TURBT or severe postoperative gross hematuria. ${ }^{11}$ After immediate instillation chemotherapy after low-risk NMIBC surgery, as the recurrence rate is very low, consolidation therapy of repeated installation is not necessary. ${ }^{8}$ Middle and high risk NMIBC requires subsequent bladder instillation chemotherapy or immunotherapy. Clinically, the relatively ideal drug for bladder instillation should be highly sensitive to cancer cells. After instillation, it can quickly penetrate the bladder wall and effective concentration of the drug is reached in the epithelial tissue, reducing the systemic absorption of the drug and causing less adverse reactions.

Through Cox multivariate regression analysis, we believe that the six selected indicators can objectively predict and evaluate the risk of recurrence of NMIBC, and can be taken as reference for the establishment of a reasonable treatment plan, providing a certain theoretical basis for clinical prediction and evaluation of the recurrence of NMIBC.

\section{References}

1. Srivastava AK, Singh PK, Singh D, et al. Evaluation of urinary XIAP as a diagnostic biomarker of carcinoma of urinary bladder. Tumor Biol. 2014, 35(8):8243-8248.

2. Ro JY, Staerkel GA, Ayala AG. Cytologic and histologic features of superficial bladder cancer. Urol Clin North Am. 1992, 19(3):435-453.
3. Konety BR, Chang SS. Management of bladder cancer:a comprehensive text with clinical scenarios. New York:Springer. 2015, 223-238.

4. Seo GH, Kim JH, Ku JH. Clinical practice pattern of immediate intravesical chemotherapy following transurethral resection of a bladder tumor in Korea: national health insurance database study. Sci Rep. 2016, 15(6):22716.

5. Carvalho DR. Improving access to adjuvant intravesicaly therapy for non-muscle invasive bladder cancer in a community hospital. Urol Nurs. 2015, 35(6):287-291.

6. Sylvester RJ, Oosterlinck W, Holmang S, et al. Systematic review and individual patient data meta-analysis of randomized trials comparing a single immediate instillation of chemotherapy after transurethral resection with transurethral resection alone in patients with stage pTa-pT1 urothelial carcinoma of the bladder:which patients benefit from the instillation? Eur Urol. 2016, 69(2):231-244.

7. Lamm DL. Intravesieal therapy for superficial bladder cancer:slow but steady progress. J Clin Oncol. 2003, 21(23):4259-4260.

8. Sylvester RJ, Oosterlinck W, van der Meijden AP. A single immediate postoperative instillation of chemotherapy decreases the risk of recurrence in patients with stage Ta, T1 bladder cancer:a metaanalysis of published results of randomized clinical trials. J Urol. 2004, 171(6 Pt 1):2186-2190.

9. Berrum-Svennung I, Granfors T, Jahnson S, et al. A single instillation of epirubicin after transurethral resection of bladder tumors prevents only small recurrences. J Urol. 2008. 179 (1):101-105.

10. Hendricksen K, Witjes WP, Idema JG, et al. Comparison of three schedules of intravesical epirubicin in patients with non-muscle-invasive bladder cancer. Eur Urol. 2008, 53(5):984-991.

11. Oddens JR, van der Meijden AP, Sylvester R. One immediate postoperative instillation of chemotherapy in low risk Ta,T1 bladder cancer patients. Is it always safe? Eur Urol. 2004, 46(3):336-338. 